\title{
Program FFlexCom - High Frequency Flexible Bendable Electronics for Wireless Communication Systems
}

\author{
Tilo Meister*, Frank Ellinger*, Johann W. Bartha*, Manfred Berroth ${ }^{\mathrm{xv}}$, Joachim Burghartz ${ }^{\|^{\mathrm{xv}}}$, Martin \\ Claus*, Lothar Frey $^{\S}$, Alessio Gagliardi ${ }^{\text {xii }}$, Marius Grundmann ${ }^{\text {xiii }}$, Jan Hesselbarth ${ }^{\mathrm{xv}}$, Hagen Klauk ${ }^{\dagger \dagger}$, Karl \\ Leo*, Paolo Lugli $^{\text {xii }}$, Stefan Mannsfeld*, Yiannos Manoli**, Renato Negra ${ }^{\ddagger \ddagger}$, Daniel Neumaier ${ }^{\mathrm{x}}$, Ullrich \\ Pfeiffer $^{\dagger}$, Thomas Riedl ${ }^{\dagger}$, Susanne Scheinert ${ }^{\text {xi }}$, Ullrich Scherf ${ }^{\dagger}$, Andreas Thiede ${ }^{\text {xiv }}$, Gerhard Troester ${ }^{\ddagger}$, Martin \\ Vossiek $^{\S}$, Robert Weigel ${ }^{\S}$, Christian Wenger ${ }^{\Uparrow}$,Golzar Alavill, Markus Becherer ${ }^{\text {xii }}$, Carlos Alvarado \\ Chavarin ", Mohammed Darwish ${ }^{\text {xii }}$, Martin Ellinger ${ }^{\S}$, Chun-Yu Fan ${ }^{\ddagger \ddagger}$, Martin Fritsch ${ }^{\dagger}$, Frank Grotjahn ${ }^{\text {xiv }}$, \\ Marco Gunia*, Katherina Haase*, Philipp Hillger ${ }^{\dagger}$, Koichi Ishida*, Michael Jank ${ }^{\S}$, Stefan Knobelspies ${ }^{\ddagger}$, \\ Matthias Kuhl**, Grzegorz Lupina ${ }^{\natural}$, Shabnam Mohammadi Naghadeh*, Niko Münzenrieder ${ }^{\ddagger}$, Sefa Özbek ${ }^{\mathrm{xv}}$, \\ Mahsa Rasteh $^{\mathrm{xv}}$, Giovanni A. Salvatore ${ }^{\ddagger}$, Daniel Schrüfer ${ }^{\S}$, Carsten Strobel*, Manuel Theisen ${ }^{\dagger}$, Christian \\ Tückmantel $^{\dagger}$, Holger von Wenckstern ${ }^{\text {xiii }}$, Zhenxing Wang $^{\mathrm{x}}$, Zhipeng Zhang ${ }^{\text {xii }}$ \\ ${ }^{*}$ TU Dresden, ${ }^{\dagger}$ Bergische Universität Wuppertal, ${ }^{\ddagger}$ ETH Zürich, ${ }^{\S}$ Friedrich-Alexander Universität Erlangen-Nürnberg,

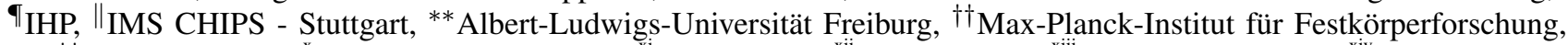 \\ ${ }_{\ddagger}^{\ddagger}$ RWTH Aachen, ${ }^{x}$ AMO GmbH - Aachen, ${ }^{x i}$ TU Ilmenau, ${ }^{x i i}$ TU München, ${ }^{\text {xiii }}$ Universität Leipzig, ${ }^{\text {xiv }}$ Universität \\ Paderborn, ${ }^{x v}$ Universität Stuttgart
}

\begin{abstract}
Today, electronics are implemented on rigid substrates. However, many objects in daily-life are not rigid - they are bendable, stretchable and even foldable. Examples are paper, tapes, our body, our skin and textiles. Until today there is a big gap between electronics and bendable daily-life items. Concerning this matter, the DFG Priority Program FFlexCom aims at paving the way for a novel research area: Wireless communication systems fully integrated on an ultra-thin, bendable and flexible piece of plastic or paper. The Program encompasses 13 projects led by 25 professors. By flexibility we refer to mechanical flexibility, which can come in flavors of bendability, foldability and, stretchability. In the last years the speed of flexible devices has massively been improved. However, to enable functional flexible systems and operation frequencies up to the sub-GHz range, the speed of flexible devices must still be increased by several orders of magnitude requiring novel system and circuit architectures, component concepts, technologies and materials.
\end{abstract}

\section{INTRODUCTION}

Electronic devices have mainly been advanced regarding integration density, performance, power consumption and costs. The speed of this development in the past decades for silicon based electronics is now expected to die soon after the year 2020 due to thermal noise. This development has long become clear and additional directions for the development of future technologies have increasingly been focused. A most interesting and new property of those future electronics is mechanical flexibility.

Mechanical flexibility can come in the flavors bendability, foldability and stretchability. It can come together with light weight, ultra-thinness, transparency, large area integration, biocompatibility and easy recyclability. These properties can be achieved by modern TOLAE (thin film organic and large area electronics) technologies. Cut-off frequencies in the $\mathrm{MHz}$ range and bending radii down to $50 \mu \mathrm{m}$ were reported for
TOLAE devices such as transistors [1]. These recent achievements point towards a totally novel and promising research area: Wireless communication systems fully integrated on ultra-thin, bendable and flexible substrates such as plastic or even paper. In this regard, the German Research Foundation (DFG) Priority Program SPP 1796 named FFlexCom [2], [3] wants to pave the way for the first circuits and systems for wireless communications, which are fully integrated in flexible TOLAE technologies. The Program started end of 2015 with 13 projects lead by 25 professors and will continue until 2022. However, for flexible systems with sufficiently high operation frequencies for wireless communications, the speed of flexible TOLAE devices and circuits will have to be massively increased.

\section{A. Four Pillars of The FFlexCom Program}

Mainly four groups of emerging materials, which are also the four pillars of the FFlexCom Program (see Fig. 1b), currently enable flexible circuits:

1) Organic semiconductors can be deposited and structured on flexible substrates with thin- and thick-film technologies. In combination with compatible conductive and dielectric materials circuits can be integrated. Especially for thick-film technologies, many substrates are possible, because no high temperature is required. Most prominent are plastic films and paper. Organic electronics at the moment tend to require large supply voltages. $60 \mathrm{~V}$ is not uncommon. Therefore, besides improving speed and stability in air, efforts have to be made to reduce the supply voltage required. With reported saturation mobilities in the range of $2 \mathrm{~cm}^{2} / \mathrm{Vs}$ and 


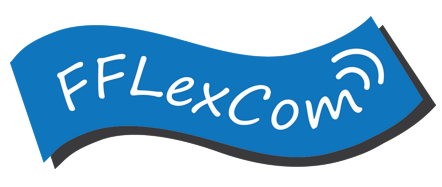

(a)

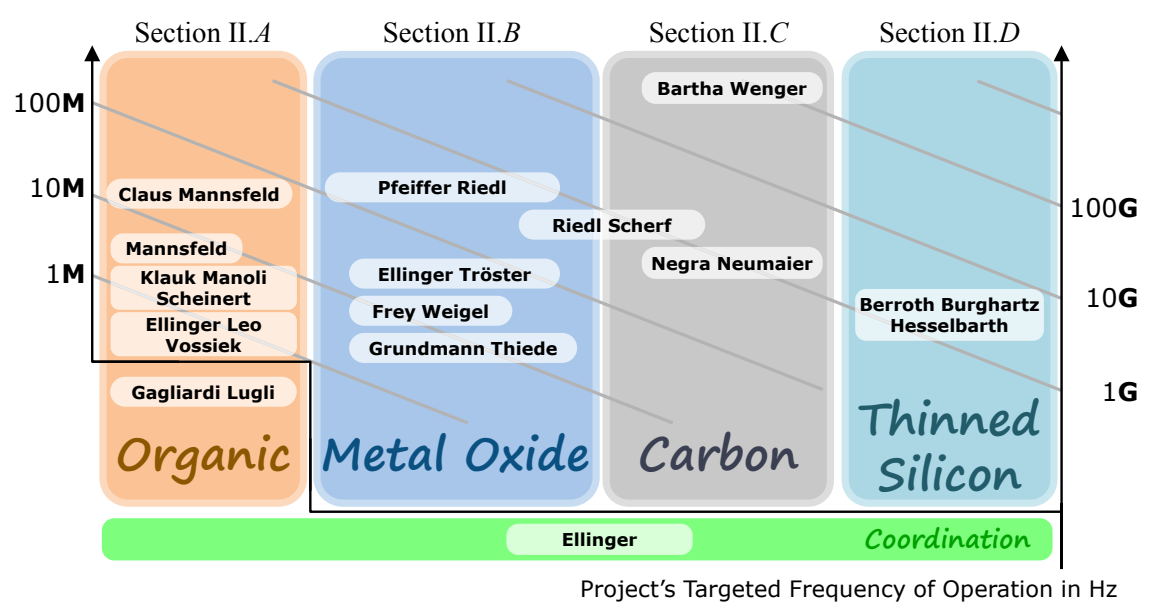

(b)

Fig. 1. (a) Logo of FFlexCom Priority Program SPP 1796 - High Frequency Flexible Bendable Electronics for Wireless Communication Systems. (b) Program pillars, organization of this paper, and project overview with their targeted frequency ranges.

transistor transit frequencies slightly above $10 \mathrm{MHz}$ they are currently the slowest pillar of flexible electronics.

2) Certain metal oxides and their alloys act as a semiconductor and can be deposited by thin-film technologies. In combination with compatible conductive and dielectric materials circuits can be integrated. Possible flexible substrates are temperature stable plastic films. These substrate materials require a trade-off between the highest process temperature and the electric performance. On the one hand, the performance (in particular the saturation mobility) is usually better for higher annealing temperatures. On the other hand, flexible substrates are increasingly instable with increasing temperatures. Saturation mobilities in the range of $20 \mathrm{~cm}^{2} / \mathrm{Vs}$ and transistor transit frequencies above $100 \mathrm{MHz}$ have been reported on polyimide films.

3) Carbon allotropes and nanowires can exhibit semiconducting properties. The growth and production of highly pure solutions of the semiconducting allotropes and nanowires has been established in the last years. However, their structuring to form transistor devices remains challenging. The carbon based devices can usually have a very small footprint. In the rigid case, the saturation mobilities of carbon nanotubes $100000 \mathrm{~cm}^{2} / \mathrm{Vs}$ and graphene $200000 \mathrm{~cm}^{2} / \mathrm{Vs}$ can by far exceed that of crystalline silicon $1400 \mathrm{~cm}^{2} / \mathrm{Vs}$. For graphene on flexible films so far saturation mobilities of above $1000 \mathrm{~cm}^{2} / \mathrm{Vs}$, which is comparable to crystalline silicon already, have been reported. These results promise transistor transit frequencies in the high $\mathrm{GHz}$-range.

4) Monocrystalline silicon circuits can be thinned down to a few tens of micrometers. The thinned chip becomes flexible and can for example be transferred to a plastic film. The result is a flexible packaged silicon circuit. While these thinned technologies can benefit from the vast experience gained in the past decades, the handling during the thinning process and packaging are still very challenging. Also, electric characteristics may change significantly during the thinning process. The transistor's transit frequencies are in the range of $100 \mathrm{GHz}$.

\section{B. Key Techniques for Advanced Device Architectures}

It is common to organic and metal oxide technologies that layer thicknesses can be very thin, down to tens of nanometers, and at the same time can be controlled precisely. However, control of lateral dimensions is much more challenging and becomes exceedingly expensive at around $1 \mu \mathrm{m}$. As a consequence self-alignment techniques, shadowing techniques, and vertical structures are becoming essential for advanced flexible organic and metal oxide devices. Self-alignment and shadowing are now frequently used to align the structures in the different layers (gate, drain, source, semiconductor in channel, etc.) of devices to be able to manufacture aggressively scaled transistors and minimize parasitics.

Vertical device architectures use a vertical channel and a vertical carrier transport such that the channel length is defined by a layer "thinness". Currently one can observe mainly two kinds of vertical structures. For the first kind, structured semiconductor layers are deposited with well defined thinnesses, which define device dimensions. Contact electrodes are fabricated above, below, and/or between those layers. Their thinnesses also define device dimensions. See Fig. $2 b$ for an example. For the second kind of vertical architectures a structured dielectric layer is deposited with a defined thinness, possibly with contact electrodes above and/or below. Against the vertical walls of the deposited dielectric layer, subsequent semiconducting/dielectric/electrode layers are deposited, again with a well defined thinness. See [4] for an example. This first kind of vertical architectures gives enhanced control over device dimensions in one spatial direction, which is usually aligned with the channel length. The second kind of vertical architectures effectively gives enhanced control over device 
dimensions in two spatial directions, usually used for precise control of channel length and dielectric thickness.

\section{Projects And Flexible Semiconductor TECHNOLgOIES OF THE FFLEXCOM PROGRAM}

This paper and the FFlexCom Priority Program are structured according to the four groups of emerging bendable technologies described above. The four pillars "Organic", "Metal Oxide", "Carbon", and "Thinned Silicon" with the 13 projects of FFlexCom are illustrated in Fig. 1b. The figure also shows the projects' targeted frequencies. Figure 1a shows the FFlexCom logo. The remainder of this paper presents the core research topics and innovations of the 13 individual projects. All of them concern bendable electronics for wireless communication systems.

\section{A. Organic Semiconductors - Fullerene, TIPS-Pentacene, etc.}

Project Gagliardi-Lugli - SIMULATION TOOLS FOR HIGH FREQUENCY FLEXIBLE ELECTRONIC DEVICES - implements and validates a multi-scale simulation framework for organic based thin-film field-effect transistors (OFETs) and thin-film carbon nanotube FETs (CNT-FETs). The framework combines models of different scales to include many known effects and thus achieves a high accuracy. The employed simulation models include drift-diffusion, kinetic Monte Carlo, and circuital equivalent. The framework can simulate different materials, contact interfaces, oxide interfaces, doping states and trap states. The impact on device performance of all the afore-mentioned effects can be predicted. Consequently, device physics can be understood well and optimized. Particularly relevant for future advancements is the new possibility to predict and optimize blended materials, which could allow operation frequencies of up to $10 \mathrm{MHz}$. These optimizations are only possible because of the unique combination of considering nano-scale effects, kinetic methods and drift diffusion models at the same time.

Project Ellinger-Leo-Vossiek - FleXART - FleXIBLe ACtIVE RADAR BACKSCATter TAG IN ORganic EleCTRONICS - develops vertical organic permeable base transistors (VO-PBT) as shown in Fig. 2a. Figure 2b shows their cross-section. They can be fabricated on flexible substrates as well as on glass substrates. On glass an $f_{\mathrm{T}}$ of $30 \mathrm{MHz}$ has been observed. The project has developed compatible passives, so that integrated flexible organic circuits are possible. Using this technology an LC-oscillator with an oscillation frequency of $5.2 \mathrm{MHz}$ was demonstrated. In the project the first localization system employing a credit-card-sized organic active radar backscatter tag using the switched injection-locked oscillator (SILO) concept will be developed and tested.

Project Klauk-Manoli-Scheinert - Megahertz ORGANIC THIN-FILM TRANSISTORS FOR FLEXIBLE BIOMEDICAL SYSTEMS - develops and improves organic TFTs and compatible passives as well as their modeling. Special focus are wireless implants with neural interfaces, small supply voltages (in the order of $3 \mathrm{~V}$ ), and low power consumption.

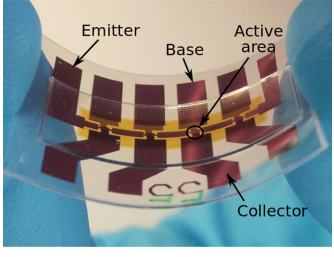

(a)

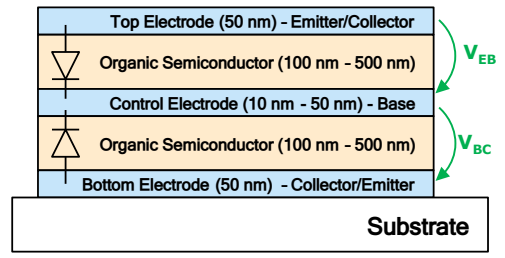

(b)
Fig. 2. (a) Flexible organic vertical permeable base transistor (VO-PBT) and (b) its cross-section [5], [6].

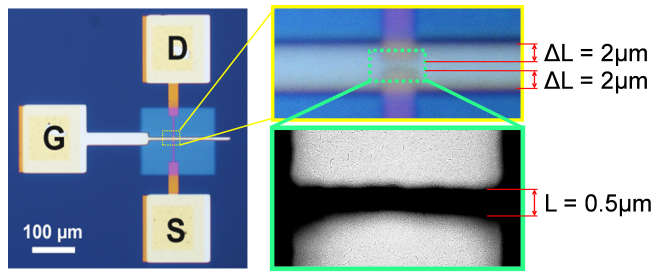

Fig. 3. Aggressively scaled lateral organic TFT (channel length $\mathrm{L}=0.5 \mu \mathrm{m}$ ).

In the project, a channel length of the organic TFTs in the submicron range was achieved (see Fig. 3). Current EDA models for OFETS are being refined based on verified FEM simulations of the devices to greatly enhance the accuracy of the compact EDA models. In particular, the modeling of the drain contact resistance has been improved. While the p-type organic TFTs are usable for signal processing and amplification, the n-type TFTs can and will only be used for biasing. Introducing the slow n-type transistors into the technology still has a great benefit. They enable a current biasing scheme, which greatly reduces the dependence of the transconductance on the transistor's threshold voltage. Thus a remarkably strong robustness against manufacturing variations is achieved on circuit design level, which is demonstrated by the project in a tunable $\mathrm{V}_{\text {th }}$-independent bandpass filter.

Project Mannsfeld - Solution-Processed, Air-STABle, ANd High-Cutoff Frequency ORganic TransisTORS FOR WIRELESS COMMUNICATION SYSTEMS - develops organic field-effect transistors that are processed from solution. The aim is to remarkably increase $f_{T}$ by combining very high mobility solution-processing technologies with short-channel device architectures, both planar and vertical. Using solution deposition strategies (e.g. solution-shearing see Figs. $4 \mathrm{a}$ and $4 \mathrm{~b}$ ) enables the controlled growth of the organic semiconductor from solution. With these strategies, highly aligned crystals can be formed in a reliable and reproducible manner, giving rise to potential large-area, lowcost manufacturing of organic TFTs. Currently, devices with an effective saturation mobility $\mu_{\text {sat }}$ between 6 and $10 \mathrm{~cm}^{2} / \mathrm{Vs}$ can reliably be fabricated in a planar device architecture.

At channel lengths of $8 \mu \mathrm{m}$ (see Fig. 4c) devices still exhibit mobilities of 1 to $2 \mathrm{~cm}^{2} /$ Vs. For these devices, transit frequencies of about $500 \mathrm{kHz}$ are estimated. Within this project, these values are expected to increase significantly due to consequent scaling of the devices dimensions and engineering of the solution deposition techniques. 


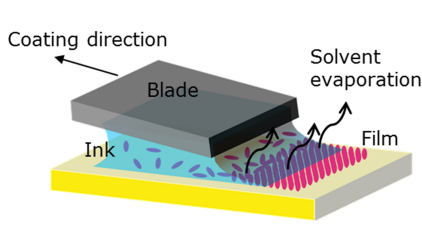

(a)

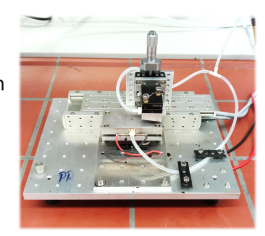

(b)

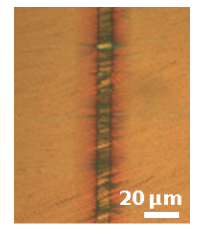

(c)
Fig. 4. (a) Schematic of shearing process used to form highly crystalline organic semiconductor film. (b) Lab set-up for solution-shearing. (c) Microscopic image of device with sheared organic semiconductor and $\mathrm{L}=8 \mu \mathrm{m}$.

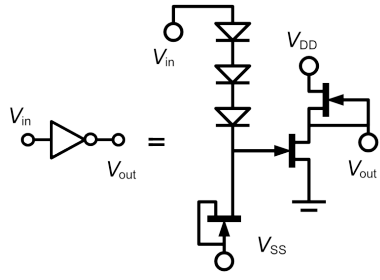

(a)

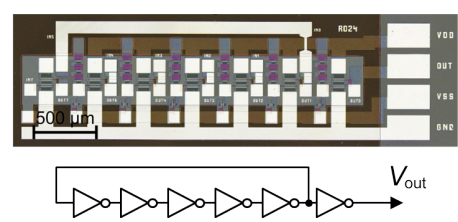

(b)
Fig. 5. (a) Schottky-diode FET logic (SDFL) inverter and (b) die photo and schematic of five stage SDFL ring oscillator using ZnO TFTs.

Project Claus-Mannsfeld - HIGH-FREQUENCY MODELING AND CHARACTERIZATION OF PRINTED ORGANIC CRYSTALLINE TRANSISTORS - develops high-frequency models for the organic technology developed in Project Mannsfeld, which is presented above. A quasi-static compact model captures the characteristics of the fabricated devices for circuit simulation. Detailed device models with special emphasis on direction dependent low field mobility and an improved trap-induced Shockley-Read-Hall (SRH) recombination model is developed to predict high frequency and non-quasi-static device performances. After validating the device models they are used to optimize the devices' saturation mobility and contact resistance by further engineering the printing process. Circuits relevant for RFIDs will serve as benchmarks and for validation during these improvements.

\section{B. Metal Oxide Semiconductors $-\mathrm{In}_{2} \mathrm{O}_{3}, \mathrm{ZnO}, \mathrm{SnO}_{2}$, etc.}

Project Grundmann-Thiede - FLEXIBLE ANALOGUE AND DIGITAL CIRCUIT BLOCKS IN AMORPHOUS METAL OXIDES explores TFTs using ternary zinc-tin oxide (ZTO) thin films. Using a combinatorial approach, it has been possible to evaluate the optical, electrical, and device properties for almost all possible cation compositions in the semiconducting layer. With this method an optimal device performance for a ratio of $\mathrm{Zn} /(\mathrm{Zn}+\mathrm{Sn}) \approx 0.6$ could be determined. The project also develops metallization layers that are used for wiring, MIM structures, and Schottky diodes. So far, using $\mathrm{ZnO}$ devices, three and five stage Schottky-diode FET logic (SDFL) ring oscillators with $110 \mathrm{~ns}$ delay per stage and an oscillation frequency of several hundred $\mathrm{kHz}$ have been demonstrated [7] (see Fig. 5). The supply voltage was up to $8 \mathrm{~V}$. With the improved ZTO layer the operating voltages will be further reduced to below $5 \mathrm{~V}$ and operating speeds in the $\mathrm{MHz}$ regime are expected.

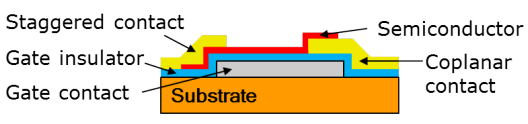

Fig. 6. Cross-section of alternating contact metal-oxide TFTs.

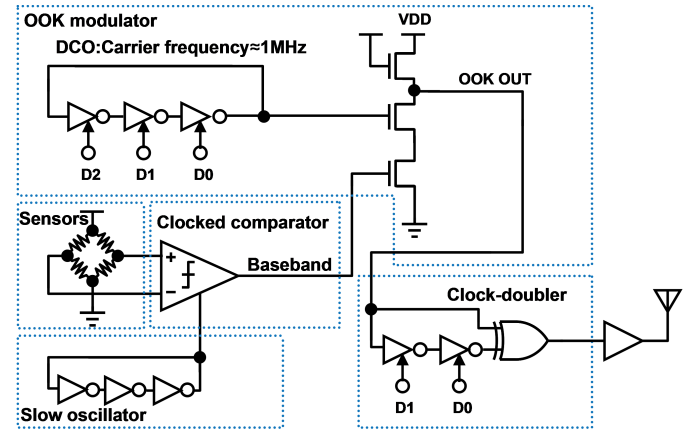

Fig. 7. System level schematic of OOK transmitter.

Project Frey-Weigel - THIN-FILM TRANSISTORS WITH NOVEL ARCHITECTURE FOR RF CIRCUITS AND SySTEMS studies alternating contact TFTs (ACTFTs, see Fig. 6). Stateof-the-art TFTs have both source and drain electrode on the same interface of the semiconducting layer. ACTFTs place them on opposite sides of the semiconducting layer, thus are not symmetric, and provide more geometric degrees of freedom. The project evaluated ACTFT devices using DC I-V and $\mathrm{C}-\mathrm{V}$ techniques, putting special emphasis on the influence of passive elements of the device architecture. $A f_{T}$ of $55 \mathrm{MHz}$ could be demonstrated for devices with a channel length of $2 \mu \mathrm{m}$. By further reducing overlap capacitances and channel lengths, the devices' $\mathrm{f}_{\mathrm{T}}$ is expected to exceed $100 \mathrm{MHz}$. In parallel, the speed of basic latches and analogue circuits is under investigation.

Project Ellinger-Tröster - WIRELESS INDIUM-GALLIUMZink-OXIDE TranSmitTers AND Devices ON MECHANiCALly-Flexible Thin-Film Substrates (W I S D $\mathrm{O} \mathrm{M})$ - investigates amorphous InGaZnO TFTs with aggressively scaled channel lengths of only $100 \mathrm{~nm}$. These short channels are structured by focused ion-beam etching. The resulting devices can be bent down to $2 \mathrm{~mm}$ radii. The project employs these devices to implement the first flexible on-off-keying (OOK) transmitter (see Fig. 7) that will be able to operate in the $13.55 \mathrm{Mhz}$ ISM band. It can be used to transmit information and sensor data such as temperature, light intensity, or humidity. Previous compact device models [8] are being advanced [9] to allow an efficient circuit design.

Project Pfeiffer-Riedl - A METAL-OXIDE TFT TECHNOLOGY PLATFORM FOR FLEXIBLE RF COMMUNICATION SYSTEMS (10BY 10.COM) - investigates self-aligned $\mathrm{ZnSnO}$ and InGaZnO based TFTs with gate lengths of down to $2 \mu \mathrm{m}$ and a gate-drain/gate-source overlap of $0.5 \mu \mathrm{m}$. New highly efficient ALD based encapsulation concepts have been developed based on [10] to improve the stability of the devices. The project was able to fabricate TFTs with a saturation mobility of 


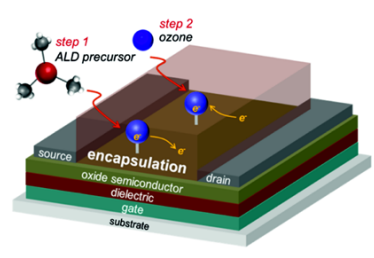

(a)

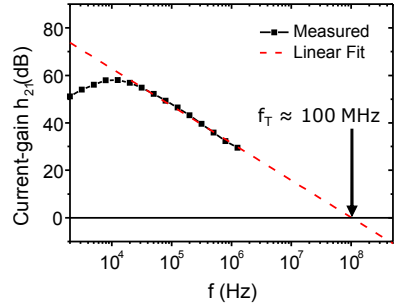

(b)
Fig. 8. (a) Cross-section with ALD based encapsulation and (b) measured $\mathrm{f}_{\mathrm{T}}$ of $2 \mu \mathrm{m}$ encapsulated InGaZnO TFT device.

above $8 \mathrm{~cm}^{2} / \mathrm{Vs}$ and to demonstrate an $\mathrm{f}_{\mathrm{T}}$ of $100 \mathrm{MHz}$ of those devices (see Fig. 8). Based on this technology circuit topologies that can operate beyond $\mathrm{f}_{\mathrm{T}}$ are investigated. For the first time a square-law detector that operates at $\mathrm{f}_{\mathrm{T}}$ of the devices has been demonstrated. It has a sensitivity of $35 \mathrm{~V} / \mathrm{W}$ at $100 \mathrm{MHz}$.

\section{Semiconducting Carbon - Graphene, Carbon Nano Tubes}

Project Riedl-Scherf - HIGH-SPEED FLEXIBLE TRANSISTORS AND TUNNEL DIODES AND CIRCUITS BASED ON THEM - develops both semiconducting metal-oxide based TFTs and single wall carbon nanotube (SWNT) based TFTs.

Metal-oxide based TFTs using different semiconducting metal-oxides are fabricated by means of atomic layer deposition (ALD). $\mathrm{In}_{2} \mathrm{O}_{3}, \mathrm{ZnO}, \mathrm{SnO}_{2}$, and $\mathrm{IZO}$ are used as semiconductor materials. The ternary compound IZO is created as nano laminates by ALD from $\mathrm{In}_{2} \mathrm{O}_{3}$ and $\mathrm{ZnO}$. Compared to the other metal-oxides it has an increased mobility and a decreased sub-threshold slope. Planar devices as well as vertical device architectures with high aspect ratios are being investigated. So far the planar n-type devices achieved a saturation mobility of $1.5 \mathrm{~cm}^{2} / \mathrm{Vs}$, on-voltages of $0 \mathrm{~V}$, subthreshold slopes of $0.5 \mathrm{~V} /$ decade and low hysteresis.

For SWNT-TFTs the semiconducting SWNTs are sorted out from metallic ones by polymer wrapping with the polyfluorene PF1-12 or P3DDT. Afterwards spin coating is used to form a SWNT network as shown in Fig. 9a which serves as channel. The cross-section of the SWNT-TFTs is illustrated in Fig. 9b. The devices exhibit the ambipolar characteristic and is shown for different channel lengths in Fig. 9c. A contact resistancecorrected charge carrier mobility of up to $50 \mathrm{~cm}^{2} / \mathrm{Vs}$ has been extracted [11].

Project Negra-Neumaier - GLECS - GRAPHENE ENABLED FLEXIBLE HIGH-FREQUENCY ELECTRONIC CIRCUITS - develops a complete process for grapheneon-foil including graphene FETs, diodes, varactors, and passives. The technology includes four metal and three dielectric layers. The carrier mobility of the graphene layer on the film was shown to be higher than $1000 \mathrm{~cm}^{2} / \mathrm{Vs}$ [12]. Device models for all components are implemented in a design-kit and validated to enable efficient circuit design. As a first validation scenario a branch-line coupler on foil (see Fig. 10a) was designed fabricated and characterized with

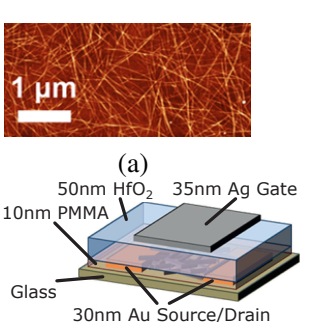

(b)

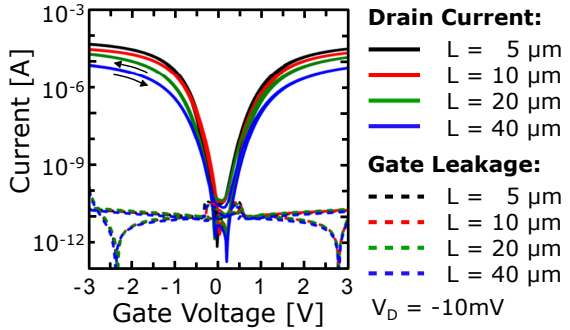

(c)
Fig. 9. (a) SEM image of SWNT network in channel. (b) Cross-section of top-gate bottom-contact transistor with spin-coated SWNT network. (c) Ambipolar transfer characteristics (channel lengths L: $5 \mu \mathrm{m}-40 \mu \mathrm{m}$ ) [11].

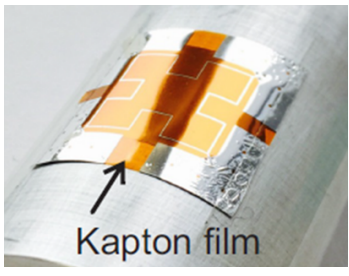

(a)

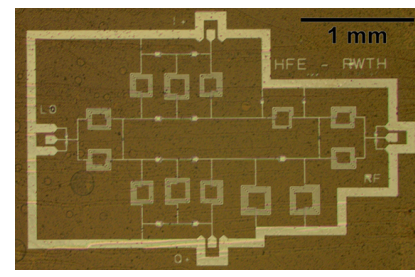

(b)
Fig. 10. (a) Branch-line coupler on Kapton film. (b) full fledged QAM receiver front-end for $2.45 \mathrm{GHz}$ ISM band using graphene diodes.

good agreements between simulation and measurements. Also a full-fledged receiver front-end using graphene diodes for the $2.45 \mathrm{GHz}$ band (see Fig. 10b) and a hall sensor on a film [13] were developed and tested.

Project Bartha-Wenger - FLEXIBLE GRAPHENE-BASE Heterojunction Transistor FOR SEVERAL HundRed GIGAHERTZ OPERATION - realizes a highly innovative vertical graphene/silicon based heterojunction transistor (GBHT). At first n-type doped amorphous hydrogenated silicon (a$\mathrm{Si}: \mathrm{H})$ was deposited on top of graphene (Gr) by means of very high frequency $(140 \mathrm{MHz})$ plasma-enhanced chemical vapor deposition (see Fig. 11b). The project demonstrated that the current (see Fig. 11a) at the (n)-a-Si:H/Gr interface is dominated by thermionic emission and recombination in the space charge region. Large Schottky diode rectification ratios of up to $1.6 \cdot 10^{4}$ (for $\pm 1 \mathrm{~V}$ ) were achieved. The Schottky barrier height, derived from temperature dependent IV characteristics, is about $0.4 \mathrm{eV}$.

In the next phase of the project, graphene will be transferred onto the surface of a-Si:H and the resulting interface will be electrically and structurally characterized. This Gr/a$\mathrm{Si}: \mathrm{H}$ interface differs from the a-Si:H/Gr interface, because graphene is transferred onto the surface of the a-Si:H substrate. Therefore both interfaces are first studied separately in order to fully understand the behavior of the final a-Si:H/Gr/a-Si:H heterojunction transistor. Operation frequencies of more than $100 \mathrm{GHz}$ are expected for the final transistor architecture.

\section{Thinned Silicon}

Project Berroth-Burghartz-Hesselbarth - A FLEXIBLE AND AdAPTIVE ENERGY-EFFICIENT HIGH-SPEED WiRELESS HUB - develops a module for wireless communication 


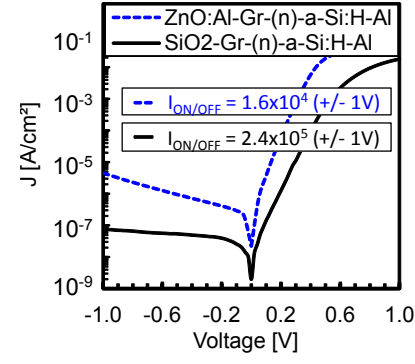

(a)

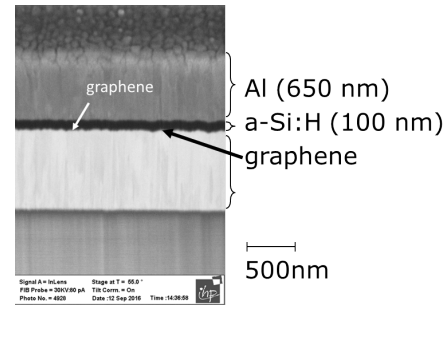

(b)
Fig. 11. (a) Current density vs. voltage of a graphene - (n)-a-Si:H junction on a $\mathrm{ZnO}: \mathrm{Al}$ substrate (blue dotted line) and on $\mathrm{SiO}_{2}$ substrate (black line) with their diode ON/OFF ratios indicated. (b) SEM image of diode test structure for (n)-a-Si:H/Gr interface with $100 \mathrm{~nm}$ of (n)-a-Si.

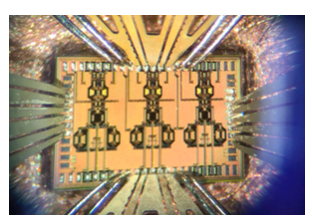

(a)

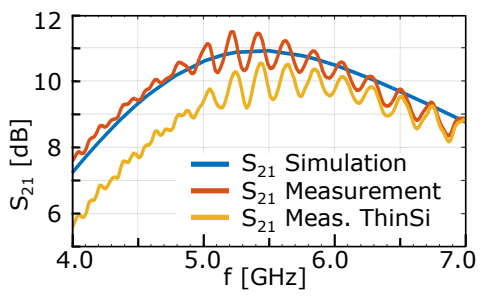

(b)
Fig. 12. Silicon BiCMOS amplifier for $5-6 \mathrm{GHz}$ thinned to $45 \mu \mathrm{m}$. (a) Die photo. (b) Simulated (blue), measured not-thinned (orange) and measured thinned (yellow) forward gain.

with an integrated antenna, using thinned $\mathrm{SiGe}$ BiCMOS chips. Circuits are thinned down after fabrication to about $20 \mu \mathrm{m}$ and are embedded in a polymer package using an innovative adaptive interconnect layout to allow a small wire pitch of below $108 \mu \mathrm{m}$ on and off chip [14]. The total package is flexible and about $100 \mu \mathrm{m}$ thick. The wireless communication module will support $100 \mathrm{Mbi} / \mathrm{s}$ and an RF output power of $10 \mathrm{~mW}$ at a carrier frequency around $5.5 \mathrm{GHz}$. Due to the mechanical flexibility the module has to adapt to changes of its shape and surroundings, i.e. it has to compensate changes of the antenna characteristics. The module uses a multiplyfed antenna, tunable load impedance, and adaptive biasing to achieve this RF adaptivity. Its final dimensions without power supply will be approximately $20 \mathrm{~mm}$ by $5 \mathrm{~mm}$ with a thickness of only $100 \mu \mathrm{m}$. Figure 12a shows a die photo of the thinned $(45 \mu \mathrm{m})$ three-path $5 \mathrm{GHz}$ to $6 \mathrm{GHz} 0.25 \mu \mathrm{m} \mathrm{SiGe}$ BiCMOS power amplifier. Figure $12 \mathrm{~b}$ shows the simulated and the measured forward gain accordingly.

\section{CONCLUSION}

In the next years, the presented projects of the German Research Foundation (DFG) Priority Program SPP 1796 FFlexCom, we will massively increase the speed of, the reliability of, and the experience with flexible electronics. It will pave the way for wireless communication with flexible electronics. The Program FFlexCom started in the end of 2015 with 13 projects and 25 leading professors and will continue until 2022. In the near future flexible devices, as those developed in this Program, will enable unforeseen applications, because they will integrate into our daily live much more naturally than rigid ones. They will not only be bendable, foldable, and strechtable, but also affordable, light weight, ultra-thin, transparent, biocompatible and easily recyclable.

\section{ACKNOWLEDGMENT}

This work and all presented projects are funded by the German Research Foundation (DFG) under Priority Program SPP 1796 - FFlexCom. Project Ellinger - Tröster is in part funded by SNF/DFG DACH under grant number 160347 . Details of the Priority Program and the involved projects can be found at [2] and [3]. The authors thank all researchers of the SPP 1796.

\section{REFERENCES}

[1] G. A. Salvatore, N. Münzenrieder, T. Kinkeldei, L. Petti, C. Zysset, I. Strebel, L. Büthe, and G. Tröster, "Wafer-scale design of lightweight and transparent electronics that wraps around hairs," Nature Communications, vol. 5, pp. 2982-, Jan. 2014.

[2] FFlexCom Programme, "FFlexCom programme website," May 2017. [Online]. Available: http://www.fflexcom.de

[3] German Research Foundation (DFG), "GEPRIS Website: SPP 1796 FFlexCom,” May 2017. [Online]. Available: http://gepris.dfg.de/gepris/ projekt $/ 255449811$ ?language $=\mathrm{en}$

[4] R. Parashkov, E. Becker, S. Hartmann, G. Ginev, D. Schneider, H. Krautwald, T. Dobbertin, D. Metzdorf, F. Brunetti, C. Schildknecht, A. Kammoun, M. Brandes, T. Riedl, H.-H. Johannes, and W. Kowalsky, "Vertical channel all-organic thin-film transistors," Applied Physics Letters, vol. 82, no. 25, pp. 4579-4580, 2003.

[5] M. P. Klinger, A. Fischer, F. Kaschura, J. Widmer, B. KheradmandBoroujeni, F. Ellinger, and K. Leo, "Organic power electronics: Transistor operation in the ka/cm(2) regime," Scientific Reports, vol. 7, pp. 44713-, Feb. 2017.

[6] M. P. Klinger, A. Fischer, F. Kaschura, R. Scholz, B. LÃ $1 / 4$ ssem, B. Kheradmand-Boroujeni, F. Ellinger, D. Kasemann, and K. Leo, "Advanced organic permeable-base transistor with superior performance," Advanced Materials, vol. 27, no. 47, pp. 7734-7739, 2015.

[7] F. J. Klüpfel, H. von Wenckstern, and M. Grundmann, "Ring oscillators based on ZnO channel JFETs and MESFETs," Advanced Electronic Materials, vol. 2, no. 7, pp. $1500431-$ n/a, 2016, 1500431.

[8] C. Perumal, K. Ishida, R. Shabanpour, B. K. Boroujeni, L. Petti, N. S. Münzenrieder, G. A. Salvatore, C. Carta, G. Tröster, and F. Ellinger, "A compact a-IGZO TFT model based on MOSFET SPICE level=3 template for analog/rf circuit designs," IEEE Electron Device Letters, vol. 34, no. 11, pp. 1391-1393, Nov 2013.

[9] R. Shabanpour, T. Meister, B. K. Boroujeni, L. Petti, N. Münzenrieder, G. A. Salvatore, G. Tröster, C. Carta, and F. Ellinger, "A transistor model for a-IGZO TFT circuit design built upon the RPI-aTFT model," in New Circuits and Systems Conference (NEWCAS), 2017, to be published.

[10] M. Fakhri, N. Babin, A. Behrendt, T. Jakob, P. Görrn, and T. Riedl, "Facile encapsulation of oxide based thin film transistors by atomic layer deposition based on ozone," Advanced Materials, vol. 25, no. 20, pp. 2821-2825, 2013.

[11] S. P. Schieß1, N. Fröhlich, M. Held, F. Gannott, M. Schweiger, M. Forster, U. Scherf, and J. Zaumseil, "Polymer-sorted semiconducting carbon nanotube networks for high-performance ambipolar field-effect transistors," ACS Applied Materials \& Interfaces, vol. 7, no. 1, pp. 682689, 2015, PMID: 25493421.

[12] Z. Wang, L. Banszerus, M. Otto, K. Watanabe, T. Taniguchi, C. Stampfer, and D. Neumaier, "Encapsulated graphene-based hall sensors on foil with increased sensitivity," physica status solidi (b), vol. 253, no. 12, pp. 2316-2320, 2016.

[13] Z. Wang, M. Shaygan, M. Otto, D. Schall, and D. Neumaier, "Flexible hall sensors based on graphene," Nanoscale, vol. 8, pp. 7683-7687, 2016.

[14] G. Alavi, H. Sailer, H. Richter, B. Albrecht, M. Alshahed, C. Harendt, and J. N. Burghartz, "Micro-hybrid system in polymer foil based on adaptive layout," in 2016 6th Electronic System-Integration Technology Conference (ESTC), Sept 2016, pp. 1-5. 